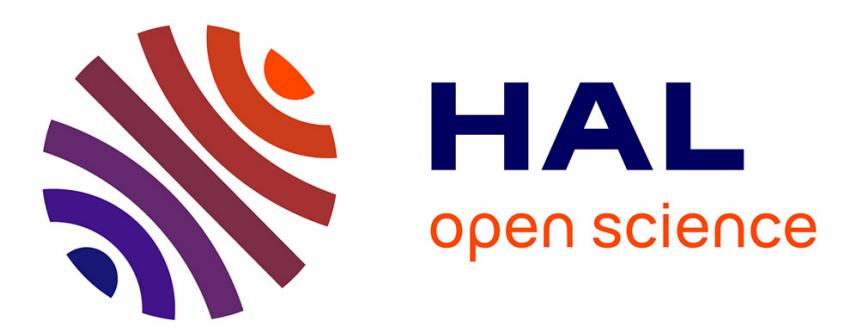

\title{
WOSD 2011 The First International Workshop on Open Systems Dependability
}

Mario Tokoro, Karama Kanoun, Kimio Kuramitsu, Jean-Charles Fabre

\section{To cite this version:}

Mario Tokoro, Karama Kanoun, Kimio Kuramitsu, Jean-Charles Fabre. WOSD 2011 The First International Workshop on Open Systems Dependability. International Workshop on Open Systems Dependability (WOSD), organised with DSN 2011, Jun 2011, Hong Kong, China. pp.593 - 594. hal-01981173

\section{HAL Id: hal-01981173 \\ https://hal.science/hal-01981173}

Submitted on 14 Jan 2019

HAL is a multi-disciplinary open access archive for the deposit and dissemination of scientific research documents, whether they are published or not. The documents may come from teaching and research institutions in France or abroad, or from public or private research centers.
L'archive ouverte pluridisciplinaire HAL, est destinée au dépôt et à la diffusion de documents scientifiques de niveau recherche, publiés ou non, émanant des établissements d'enseignement et de recherche français ou étrangers, des laboratoires publics ou privés. 


\section{WOSD 2011 The First International Workshop on Open Systems Dependability}

\author{
Mario Tokoro \\ Sony CSL, Inc. \\ Tokyo, Japan \\ mario.tokoro@csl.sony.co.jp
}

\author{
Karama Kanoun \\ LAAS CNRS \\ Toulouse, France \\ karama.kanoun@laas.fr
}

\author{
Kimio Kuramitsu \\ Yokohama National \\ University, Japan \\ kimio@ynu.ac.jp
}

\author{
Jean-Charles Fabre \\ LAAS_CNRS \\ Toulouse, France \\ jean-charles.fabre@laas.fr
}

\section{Introduction}

Modern computer systems are increasing in complexity, spread, and scale in order to meet the diverse and sophisticated needs of the users. In the development of these systems, we inevitably use legacy codes and offthe shelf software as black box software in order to shorten the development time and lower the development cost. These systems are often connected via a network to utilize services provided by other systems, whereas services and network performance may change while in operation. We often need to change the specification and implementation of a system due to the changes of environments and users' requirements. In addition, threats caused by viruses and unauthorized accesses have to be properly removed. Therefore, modern computer systems inherently involve incompleteness of specifications and implementations and uncertainty of environments and requirements.

A system whose function, construction, and boundary keep changing over time is called an open system in contrast to a closed system whose function, construction, and boundary stay the same through the life of the system. As we see in the above discussion, the modern computer systems hold the characteristics of open systems. Thus, we need to establish new concepts, processes, architectures, and tools that attain dependability throughout the lifecycle of an open system.

Open Systems Dependability can tentatively be defined as follows: Functions, constructions, environments and boundaries of modern computer systems change over time. Hence incompleteness and uncertainty are factors that may result in failures in the future. Open Systems Dependability is the ability to continuously prevent the said factors from causing failure, to take appropriate and quick action when failures occur to minimize the damage, to safely and continuously provide the services expected by users as much as possible, and to maintain accountability for the system operations and processes.
Open Systems Dependability does not conflict with the dependability that has been studied by many researchers. Open Systems Dependability puts emphasis on providing services continuously by managing unpredictable failures on the ever-changing systems, where managing means to assure sustainability of services with the best effort, which is considered to be most important to both users and service providers.

\section{This Year's Workshop}

This new workshop aims to foster discussions about new concepts, processes, architectures and tools for Open Systems Dependability. One common theme underlying the papers accepted in this year's workshop is gaining confidence in the emerging concept of Open Systems Dependability.

We start with a keynote speech by Alexander Romanovsky, from Newcastle University. Alexander's talk, entitled "Dealing with uncertainty to ensure open system dependability", is about current trends in ICT clearly showed that the vast majority of computer-based systems of the future will be open. This makes it necessary to identify the challenge of dealing with uncertainty while ensuring dependability of open systems. In his keynote speech, he discuss various sources of uncertainty and show how the move from developing closed systems to developing open systems changes the way system dependability can be achieved. Of a particular interest to our community is the uncertainty of the erroneous system behavior, which calls for new approaches to fault tolerance. The talk will demonstrate several solutions already developed in his team for understanding, benchmarking and modeling uncertainty, as well as for architecting open systems that can tolerate uncertain behavior in their components and communication media by employing advanced recovery mechanisms.

The keynote speech is followed by 5 papers and 1 short invited paper, organized in 2 Sessions, that represent an emerging collection of ideas in the Open Systems Dependability. 
The first session is about "Application" issues for Open System Dependability.

In the first paper, "On Finding Vestiges of Internet Backbone Failures for Optimizing Wide Area Data Replication", Hirotake Abe, Hirotaka Moriya and Kyoji Umemura from Osaka University, Japan, provides some metrics based on real data collected on Internet communication failures. In their paper, a statistical outliner detection method on graph theoretic metrics is applied to pick up suspicious cases within AS-level topology data. Their finding is that the number of nodes and average clustering coefficient are promising components.

In the second paper, "Performance Modeling and Benchmarking of Bank Intermediary Business on HighPerformance Fault-Tolerant Computers", by Bo Li, Haiying Zhou, Decheng Zuo, Zhan Zhang, Peng Zhou and Long Jia, from School of computer Science and technology, Harbin Institute of Technology, Harbin, China, focuses on the bank intermediary business application. In order to evaluate Online Transaction Processing (OLTP) performance of high-performance fault-tolerant computers, the BIB benchmark is newly defined on top of database model and transaction/frame model to evaluate the performance.

"Composite Dependability Modeling for In-vehicle Networks", Hiroyuki Okamura, Tadashi Dohi, Shin'ichi Shiraishi and Mutsumi Abe from Hiroshima University, Japan, presents on an innovative approach to the dependability assessment of in-vehicle applications. The key part of their proposal is the translation from UML and SysML to a stochastic model checker. They also present an illustrative example on the dependability modeling of an auto-cruise system in the design phase.

The second section is dedicated to "System and Architecture".

The session starts with an invited talk, "Architecting Resilient Computer Systems: Overall Approach and Open Issues" by Jean-Charles Fabre from LAAS-CNRS, which shows a methodology to provide adaptation mechanisms meant to ensure dependability while coping with various modifications of applications' and system's context. To that aim, the paper proposes a representation of dependability-related attributes that may evolve during system lifecycle, and shows the usefulness of the proposed representation by showing adaptation of dependability mechanisms at runtime.
In the second paper, "Fault Model of Foreign Function Interface across Different Domains" by Shinpei Nakata, Midori Sugaya and Kimio Kuramitsu from Yokohama National University, Japan, presents a fault model for foreign function interface (FFI) that connects different two languages or software domains. Their proposed approach is implanted in D-Script, which is running on a DEOS runtime environment.

The second session's last paper entitled "Enforcing Dependable Operations by Model Checking a Virtualization Layer" by Shuichi Oikawa from Tsukuba University, Japan, addresses the provision of basic runtime layers for resilient systems by means of virtualization technologies. The paper also includes an interesting approach to the extended use of Linux Kernel module model checker for the verification of virtual machine monitor.

The workshop finishes with a panel session with all the workshop attendees. The session starts with an interesting position paper, entitled "Open Systems Dependability", by Mario Tokoro, Computer Science Laboratory, Sony. We expect the discussion around these papers to identify open problems and fundamental issues in Open System Dependability that increasingly need research and bring forward innovative ideas to the mission-critical industries.

\section{Acknowledgement}

We are thankful to all the authors who submitted papers for this first edition of the workshop and also to the DSN organization for their support. It is worth noting that the workshop was partially supported by the JST/CREST "Dependable Embedded Operating System for Practical Use" Program.

Last but not least, we are very grateful to the members of the Program Committee who contributed to the organization of this workshop, namely: Michel Cukier (University of Maryland, USA), Tadashi Dohi (Hiroshima University, Japan), Zbigniew Kalbarczyk (UIUC, USA), Kenji Kono (Keio University, Japan) Miroslaw Malek (Humboldt-Universität zu Berlin, Germany), Eliane Martins (Univ. of Campinas, Brazil) Takashi Nanya (Canon Inc., Japan), Alexander Romanovsky (Newcastle University, UK), Juan-Carlos Ruiz-Garcia (Univ. Politecnica de Valencia, Spain), Rick Schlichting (AT\&T Labs, USA), Francois Taiani (Lancaster University, UK), Jianjun Zhao (Shanghai Jiao Tong University, China). 